\title{
ON PHASE TRANSITIONS OF NUCLEAR MATTER IN THE NAMBU-JONA-LASINIO MODEL
}

\author{
TRAN HUU PHAT \\ Vietnam Atomic Energy Commission \\ LE VIET HOA \\ Hanoi University of Education \\ NGUYEN VAN LONG \\ Gia Lai Teacher College \\ NGUYEN TUAN ANH \\ Electronics Power University \\ NGUYEN VAN THUAN \\ Hanoi University of Education
}

\begin{abstract}
Within the Cornwall - Jackiw - Tomboulis (CJT) approach a general formalism is established for the study of asymmetric nuclear matter (ANM) described by the Nambu-Jona-Lasinio (NJL) model. Restricting to the double-bubble approximation (DBA)we determine the bulk properties of ANM, in particular, the density dependence of the nuclear symmetry energy, which is in good agreement with data of recent analyses.
\end{abstract}

\section{INTRODUCTION}

It is known that one of the most important thrusts of modern nuclear physics is the use of high energy heavy-ion reactions for studying the properties of excited nuclear matter and finding the evidence of nuclear phase transition between different thermodynamical states at finite temperature and density. Numerous experimental analyses indicate that there is dramatic change in the reaction mechanism for excited energy per nucleon in the interval $E^{*} / A \sim 2-5 \mathrm{MeV}$, consistently corresponding to a first or second order liquid-gas phase transition of nuclear matter [1], [2]. In parallel to experiments, a lot of theoretical papers has been published [3], [4], [5], among them, perhaps, the research based on simplified models of strongly interacting nucleons is of great interest for understanding nuclear matter under different conditions.

In this respect, this paper aims at considering nuclear phase transition in the NJL model. Here we use the CJT effective action formalism and the numerical calculation is carried out in the HF approximation. The rest of this paper is organized as follows. In Sect.II we derive the CJT effective potential and then establish the expression for binding energy per nucleon. The numerical computation is performed in Sect.III. After fixing the model parameters we determine the density dependence of Nuclear Symmetry Energy. The Sect.IV is devoted to conclusions and outlook.

\section{CJT EFFECTIVE POTENTIAL}

Let us begin with the nuclear matter modeled by the Lagrangian density:

$$
£=\bar{\psi}(i \hat{\partial}-M) \psi+\frac{G_{\sigma}}{2}(\bar{\psi} \psi)^{2}-\frac{G_{\omega}}{2}\left(\bar{\psi} \gamma^{\mu} \psi\right)^{2}+\frac{G_{\rho}}{2}\left(\bar{\psi} \vec{\tau} \gamma^{\mu} \psi\right)^{2} .
$$


Here $\psi(x)$ is the nucleon field, M the nucleon mass, $\vec{\tau}$ denotes the isospin matrices, and $G_{\sigma, \omega, \rho}$ are coupling constants.

By bosonization

$$
\check{\sigma}=\frac{g_{\sigma}}{m_{\sigma}^{2}} \bar{\psi} \psi, \check{\omega}_{\mu}=\frac{g_{\omega}}{m_{\omega}^{2}} \bar{\psi} \gamma_{\mu} \psi, \vec{\rho}_{\mu}=\frac{g_{\rho}}{m_{\rho}^{2}} \bar{\psi} \vec{\tau} \gamma_{\mu} \psi
$$

(1) takes the form

$$
\begin{aligned}
£= & \bar{\psi}(i \hat{\partial}-M) \psi+g_{\sigma} \bar{\psi} \check{\sigma} \psi-g_{\omega} \bar{\psi} \gamma^{\mu} \breve{\omega}_{\mu} \psi+g_{\rho} \bar{\psi} \gamma^{\mu} \vec{\tau} . \vec{\rho}_{\mu} \psi \\
& -\frac{m_{\sigma}^{2}}{2} \check{\sigma}^{2}+\frac{m_{\omega}^{2}}{2} \check{\omega}^{\mu} \check{\omega}_{\mu}-\frac{m_{\rho}^{2}}{2} \overrightarrow{\tilde{\rho}}^{\mu} \overrightarrow{\tilde{\rho}}_{\mu},
\end{aligned}
$$

in which $G_{\sigma, \omega, \rho}=g_{\sigma, \omega, \rho}^{2} / m_{\sigma, \omega, \rho}^{2}$.

According to $[6,7]$ we obtain the expression for the CJT effective action

$$
\begin{aligned}
V & =\frac{m_{\sigma}^{2}}{2} \sigma^{2}-\frac{m_{\omega}^{2}}{2} \omega^{2}+\frac{m_{\rho}^{2}}{2} \rho^{2} \\
& -i \int \frac{d^{4} q}{(2 \pi)^{4}} \operatorname{tr}\left[\ln S_{0}^{-1}(q) S^{p}(q)-S_{0}^{p-1}(q ; \sigma, \omega, \rho) S^{p}(q)+1\right] \\
& -i \int \frac{d^{4} q}{(2 \pi)^{4}} \operatorname{tr}\left[\ln S_{0}^{-1}(q) S^{n}(q)-S_{0}^{n-1}(q ; \sigma, \omega, \rho) S^{n}(q)+1\right] \\
& +\frac{i}{2} \int \frac{d^{4} q}{(2 \pi)^{4}} \operatorname{tr}\left[\ln C_{0}^{-1} C(q)-C_{0}^{-1} C(q)+1\right] \\
& +\frac{i}{2} \int \frac{d^{4} q}{(2 \pi)^{4}} \operatorname{tr}\left[\ln D_{0}^{\mu \nu-1} D_{\mu \nu}(q)-D_{0}^{\mu \nu-1} D_{\mu \nu}(q)+1\right] \\
& +\frac{i}{2} \int \frac{d^{4} q}{(2 \pi)^{4}} \operatorname{tr}\left[\ln R_{0}^{33 \mu \nu-1} R_{33 \mu \nu}(q)-R_{0}^{33 \mu \nu-1} R_{33 \mu \nu}(q)+1\right] \\
& -\frac{i}{2} g_{\sigma} \int \frac{d^{4} q}{(2 \pi)^{4}} \frac{d^{4} k}{(2 \pi)^{4}} \operatorname{tr}\left\{\left[S^{p}(q) \Gamma^{p}(q, k-q) S^{p}(k)+S^{n}(q) \Gamma^{n}(q, k-q) S^{n}(k)\right] C(k-q)\right\} \\
& +\frac{i}{2} g_{\omega} \int \frac{d^{4} q}{(2 \pi)^{4}} \frac{d^{4} k}{(2 \pi)^{4}} \operatorname{tr}\left\{\gamma^{\mu}\left[S^{p}(q) \Gamma^{p \nu}(q, k-q) S^{p}(k)+S^{n}(q) \Gamma^{n \nu}(q, k-q) S^{n}(k)\right] D_{\mu \nu}(k-q)\right\} \\
& -\frac{i}{4} g_{\rho} \int \frac{d^{4} q}{(2 \pi)^{4}} \frac{d^{4} k}{(2 \pi)^{4}} \operatorname{tr}\left\{\gamma^{\mu}\left[S^{p}(q) \Gamma^{p 3 \nu}(q, k-q) S^{p}(k)-S^{n}(q) \Gamma^{n 3 \nu}(q, k-q) S^{n}(k)\right] R_{33 \mu \nu}(k-q)\right\}
\end{aligned}
$$

where $\Gamma, \Gamma^{\mu}$ and $\Gamma^{3 \mu}$ are the effective vertices taking into account all higher loops contributions;

$$
\begin{aligned}
i S_{0}^{-1}(k) & =\hat{k}-M, \\
i S_{0}^{p-1}(k ; \sigma, \omega, \rho) & =i S_{0}^{-1}(k)+g_{\sigma} \sigma-g_{\omega} \gamma^{0} \omega+\frac{g_{\rho}}{2} \gamma^{0} \rho, \\
i S_{0}^{n-1}(k ; \sigma, \omega, \rho) & =i S_{0}^{-1}(k)+g_{\sigma} \sigma-g_{\omega} \gamma^{0} \omega-\frac{g_{\rho}}{2} \gamma^{0} \rho, \\
i C_{0}^{-1} & =-m_{\sigma}^{2}, \\
i D_{0 \mu \nu}^{-1} & =g_{\mu \nu} m_{\omega}^{2}, \\
i R_{033 \mu \nu}^{-1} & =-\delta_{33} g_{\mu \nu} m_{\rho}^{2},
\end{aligned}
$$


$S, C, D_{\mu \nu}$ and $R_{33 \mu \nu}$ are the propagators of nucleon, sigma, omega and rho mesons, respectively; $\sigma, \omega$ and $\rho$ are expectation values of the sigma, omega and rho fields in the ground state of ANM,

$$
\sigma=\langle\check{\sigma}\rangle=\text { const. },\langle\check{\omega}\rangle=\omega \delta_{0 \mu},\langle\check{\rho}\rangle=\rho \delta_{3 a} \delta_{0 \mu} .
$$

The ground state corresponds to the solution of

$$
\begin{aligned}
& \frac{\delta V}{\delta \phi}=0, \\
& \frac{\delta V}{\delta G}=0 .
\end{aligned}
$$

(3) is the gap equation and (4) is the Schwinger-Dyson (SD) equation for propagators G.

In this paper, we restrict ourselves to the double-bubble approximation (DBA), in which $M_{\sigma}=m_{\sigma}, M_{\omega}=m_{\omega}, M_{\rho}=m_{\rho}$. Then, after some algebra we get the expression for $\mathrm{V}$

$$
\begin{aligned}
V\left(M^{*}, \mu, T\right) & =\frac{m_{\sigma}^{2}}{2} \sigma^{2}-\frac{m_{\omega}^{2}}{2} \omega^{2}+\frac{m_{\rho}^{2}}{2} \rho^{2}+\frac{1}{\pi^{2}} \int_{0}^{\infty} q^{2} d q\left[T \ln \left(n_{q}^{p *-} n_{q}^{p *+}\right)+T \ln \left(n_{q}^{n *-} n_{q}^{n *+}\right)\right] \\
& +\frac{G_{\sigma}-2 G_{\omega}-G_{\rho} / 2}{8 \pi^{4}}\left[\int_{0}^{\infty} q^{2} d q\left(n_{q}^{p *-}-n_{q}^{p *+}\right)\right]^{2} \\
& +\frac{G_{\sigma}-2 G_{\omega}+G_{\rho} / 2}{8 \pi^{4}}\left[\int_{0}^{\infty} q^{2} d q\left(n_{q}^{n *-}-n_{q}^{n *+}\right)\right]^{2} \\
& +\frac{G_{\sigma}+4 G_{\omega}-G_{\rho}}{8 \pi^{4}}\left[\int_{0}^{\infty} q^{2} d q \frac{M^{p *}}{E_{q}^{p *}}\left(n_{q}^{p *-}+n_{q}^{p *+}\right)\right]^{2} \\
& +\frac{G_{\sigma}+4 G_{\omega}+G_{\rho}}{8 \pi^{4}}\left[\int_{0}^{\infty} q^{2} d q \frac{M^{n *}}{E_{q}^{n *}}\left(n_{q}^{n *-}+n_{q}^{n *+}\right)\right]^{2}
\end{aligned}
$$

Here $n_{k}^{a *}, a=\{p, n\}$ are the Fermi distribution function

$$
n_{k}^{a * \pm}=\frac{1}{e^{\left(E_{k}^{a *} \pm \mu^{a *}\right) / T}+1}
$$

in which

$$
\begin{aligned}
& E_{k}^{* a}=\sqrt{k^{* 2}+M^{a * 2}}, \\
& \mu^{p *}= \mu^{p}-\frac{1}{\pi^{2}}\left[G_{\omega}+\frac{G_{\rho}}{4}\right] \int_{0}^{\infty} q^{2} d q\left(n_{q}^{n *-}-n_{q}^{n *+}\right) \\
&- \frac{1}{4 \pi^{2}}\left[-G_{\sigma}+6 G_{\omega}-\frac{G_{\rho}}{2}\right] \int_{0}^{\infty} q^{2} d q\left(n_{q}^{p *-}-n_{q}^{p *+}\right), \\
& \mu^{n *}= \mu^{n}-\frac{1}{\pi^{2}}\left[G_{\omega}+\frac{G_{\rho}}{4}\right] \int_{0}^{\infty} q^{2} d q\left(n_{q}^{p *-}-n_{q}^{p *+}\right) \\
&-\frac{1}{4 \pi^{2}}\left[-G_{\sigma}+6 G_{\omega}-3 \frac{G_{\rho}}{2}\right] \int_{0}^{\infty} q^{2} d q\left(n_{q}^{n *-}-n_{q}^{n *+}\right), \\
& M^{p *}= M+\Sigma_{s}^{p}=M-\frac{1}{\pi^{2}} G_{\sigma} \int_{0}^{\infty} q^{2} d q \frac{M^{n *}}{E_{q}^{n *}}\left(n_{q}^{n *-}+n_{q}^{n *+}\right) \\
&-\frac{1}{4 \pi^{2}}\left[5 G_{\sigma}+4 G_{\omega}+G_{\rho}\right] \int_{0}^{\infty} q^{2} d q \frac{M^{p *}}{E_{q}^{p *}}\left(n_{q}^{p *-}+n_{q}^{p *+}\right),
\end{aligned}
$$




$$
\begin{aligned}
M^{n *} & =M+\Sigma_{s}^{n}=M-\frac{1}{\pi^{2}} G_{\sigma} \int_{0}^{\infty} q^{2} d q \frac{M^{p *}}{E_{q}^{p *}}\left(n_{q}^{p *-}+n_{q}^{p *+}\right) \\
& -\frac{1}{4 \pi^{2}}\left[5 G_{\sigma}+4 G_{\omega}-G_{\rho}\right] \int_{0}^{\infty} q^{2} d q \frac{M^{n *}}{E_{q}^{n *}}\left(n_{q}^{n *-}+n_{q}^{n *+}\right) .
\end{aligned}
$$

Starting from (5) we establish successively the expressions for the thermodynamical potential $\Omega$, the energy density $\epsilon$ and the binding energy per nucleon $\epsilon_{\text {bind. }}$ :

$$
\begin{array}{ll}
\text { a/ } & \Omega=V-V_{v a c}, \\
& \text { with } V_{v a c}=V(M, \rho=0, T=0) . \\
\text { b/ } & \epsilon=\Omega+\mu_{p} \rho_{p}+\mu_{n} \rho_{n}, \\
\text { c/ } & \epsilon_{\text {bind. }}=-M+\epsilon / \rho_{B}
\end{array}
$$

with

$$
\rho_{B}=\rho_{p}+\rho_{n}=\frac{1}{3 \pi^{2}}\left(k_{F_{p}}^{3}+k_{F_{n}}^{3}\right)
$$

is baryon density, and $\rho_{p}$ and $\rho_{n}$ are proton and neutron densities, respectively. It is obvious that all necessary information on dynamics of our system are provided by the formulae (5)-(9).

\section{NUMERICAL COMPUTATIONS}

At $T=0$ Eqs.(5)-(9) are respectively reduced to

$$
\begin{aligned}
& V\left(M^{*}, \mu, 0\right)=\frac{1}{8 \pi^{4}} G_{\sigma}\left[M^{p *}\left(\mu^{p *} \sqrt{\mu^{p * 2}-M^{p * 2}}-M^{p * 2} \ln \left|\frac{\mu^{p *}+\sqrt{\mu^{p * 2}-M^{p * 2}}}{M^{p *}}\right|\right)\right. \\
& \left.+M^{n *}\left(\mu^{n *} \sqrt{\mu^{n * 2}-M^{n * 2}}-M^{n * 2} \ln \left|\frac{\mu^{n *}+\sqrt{\mu^{n * 2}-M^{n * 2}}}{M^{n *}}\right|\right)\right]^{2} \\
& +\frac{1}{18 \pi^{4}} G_{\omega}\left(k_{F_{p}}^{3}+k_{F_{n}}^{3}\right)^{2}-\frac{1}{72 \pi^{4}} G_{\rho}\left(k_{F_{p}}^{3}-k_{F_{n}}^{3}\right)^{2} \\
& -\frac{1}{72 \pi^{4}}\left(G_{\sigma}-2 G_{\omega}\right)\left(k_{F_{p}}^{6}+k_{F_{n}}^{6}\right)+\frac{1}{72 \pi^{4}} \frac{G_{\rho}}{2}\left(k_{F_{p}}^{6}-k_{F_{n}}^{6}\right) \\
& +\frac{1}{8 \pi^{2}}\left[\mu^{p *}\left(2 \mu^{p * 2}-M^{p * 2}\right) \sqrt{\mu^{p * 2}-M^{p * 2}}-M^{p * 4} \ln \left|\frac{\mu^{p *}+\sqrt{\mu^{p * 2}-M^{p * 2}}}{M^{p *}}\right|\right. \\
& \left.+\mu^{n *}\left(2 \mu^{n * 2}-M^{n * 2}\right) \sqrt{\mu^{n * 2}-M^{n * 2}}-M^{n * 4} \ln \left|\frac{\mu^{n *}+\sqrt{\mu^{n * 2}-M^{n * 2}}}{M^{n *}}\right|\right] \\
& -\frac{1}{32 \pi^{4}}\left(G_{\sigma}+4 G_{\omega}+G_{\rho}\right)\left[M^{p *}\left(\mu^{p *} \sqrt{\mu^{p * 2}-M^{p * 2}}-M^{p * 2} \ln \left|\frac{\mu^{p *}+\sqrt{\mu^{p * 2}-M^{p * 2}}}{M^{p *}}\right|\right)\right]^{2} \\
& -\frac{1}{32 \pi^{4}}\left(G_{\sigma}+4 G_{\omega}-G_{\rho}\right)\left[M^{n *}\left(\mu^{n *} \sqrt{\mu^{n * 2}-M^{n * 2}}-M^{n * 2} \ln \left|\frac{\mu^{n *}+\sqrt{\mu^{n * 2}-M^{n * 2}}}{M^{n *}}\right|\right)\right]^{2} \\
& -\mu \rho_{B} \text {. }
\end{aligned}
$$




$$
\begin{aligned}
& \mu^{p *}=\mu^{p}-\frac{1}{4 \pi^{2}}\left[6 G_{\omega}-G_{\sigma}-\frac{G_{\rho}}{2}\right] \frac{\left(\mu^{p * 2}-M^{p * 2}\right)^{3 / 2}}{3} \\
& -\frac{1}{\pi^{2}}\left[G_{\omega}+\frac{G_{\rho}}{4}\right] \frac{\left(\mu^{n * 2}-M^{n * 2}\right)^{3 / 2}}{3}, \\
& \mu^{n *}=\mu^{n}-\frac{1}{4 \pi^{2}}\left[6 G_{\omega}-G_{\sigma}-3 \frac{G_{\rho}}{2}\right] \frac{\left(\mu^{n * 2}-M^{n * 2}\right)^{3 / 2}}{3} \\
& -\frac{1}{\pi^{2}}\left[G_{\omega}+\frac{G_{\rho}}{4}\right] \frac{\left(\mu^{p * 2}-M^{p * 2}\right)^{3 / 2}}{3} \\
& M^{p *}=M-\frac{1}{8 \pi^{2}}\left[5 G_{\sigma}+4 G_{\omega}+G_{\rho}\right] M^{p *}\left(\mu^{p *} \sqrt{\mu^{p * 2}-M^{p * 2}}-M^{p * 2} \ln \left|\frac{\mu^{p *}+\sqrt{\mu^{p * 2}-M^{p * 2}}}{M^{p *}}\right|\right) \\
& -\frac{1}{2 \pi^{2}} G_{\sigma} M^{n *}\left(\mu^{n *} \sqrt{\mu^{n * 2}-M^{n * 2}}-M^{n * 2} \ln \left|\frac{\mu^{n *}+\sqrt{\mu^{n * 2}-M^{n * 2}}}{M^{n *}}\right|\right), \\
& M^{n *}=M-\frac{1}{8 \pi^{2}}\left[5 G_{\sigma}+4 G_{\omega}-G_{\rho}\right] M^{n *}\left(\mu^{n *} \sqrt{\mu^{n * 2}-M^{n * 2}}-M^{n * 2} \ln \left|\frac{\mu^{n *}+\sqrt{\mu^{n * 2}-M^{n * 2}}}{M^{n *}}\right|\right) \\
& -\frac{1}{2 \pi^{2}} G_{\sigma} M^{p *}\left(\mu^{p *} \sqrt{\mu^{p * 2}-M^{p * 2}}-M^{p * 2} \ln \left|\frac{\mu^{p *}+\sqrt{\mu^{p * 2}-M^{p * 2}}}{M^{p *}}\right|\right),
\end{aligned}
$$

The masses of nucleon and mesons are chosen to be $M=939 \mathrm{MeV}, m_{\sigma}=550 \mathrm{MeV}$, $m_{\omega}=783 \mathrm{MeV}$ and $m_{\rho}=770 \mathrm{MeV}$.

The numerical calculation therefore is ready to be carried out step by step as follows. We first fix the coupling constants $G_{\sigma}$ and $G_{\omega}$. To this end, Eq.(17) or (18) is solved numerically for symmetric nuclear matter $\left(G_{\rho}=0\right)$. Its solution is then substituted into the nuclear binding energy $\epsilon_{\text {bind }}$ in (12) with $V$ given in (14), $\rho_{B}$ given in (13). Two parameters $g_{\sigma}$ and $g_{\omega}$ are adjusted to yield the the binding energy $E_{\text {bind }}=-15.8 \mathrm{MeV}$ at normal density $\rho_{B}=\rho_{0}=0.16 \mathrm{fm}^{-3}$ as is shown in Fig. 1. The corresponding values for $G_{\sigma}$ and $G_{\omega}$ are $G_{\sigma}=195.6 / M^{2}$ and $G_{\omega}=1.21 G_{\sigma}$.

As to fixing $G_{\rho}$ let us employ the expansion of nuclear symmetry energy (NSE) around $\rho_{0}$

$$
E_{\text {sym }}=a_{4}+\frac{L}{3}\left(\frac{\rho_{B}-\rho_{0}}{\rho_{0}}\right)+\frac{K_{\text {sym }}}{18}\left(\frac{\rho_{B}-\rho_{0}}{\rho_{0}}\right)^{2}+\ldots
$$

with $a_{4}$ being the bulk symmetry parameter of the Weiszaecker mass formula, experimentally we know $a_{4}=30-35 \mathrm{MeV} ; L$ and $K_{\text {sym }}$ related respectively to slope and curvature of NSE at $\rho_{0}$

$$
\begin{aligned}
L & =3 \rho_{0}\left(\frac{\partial E_{\text {sym }}}{\partial \rho_{B}}\right)_{\rho_{B}=\rho_{0}}, \\
K_{\text {sym }} & =9 \rho_{0}^{2}\left(\frac{\partial^{2} E_{\text {sym }}}{\partial \rho_{B}^{2}}\right)_{\rho_{B}=\rho_{0}} .
\end{aligned}
$$

Then $G_{\rho}$ is fitted to give $a_{4}=32 \mathrm{MeV}$, its value is $G_{\rho}=0.972 G_{\sigma}$. Thus, all of the model parameters are known. Let us now determine the density dependence of NSE. Carrying out the numerical computation with the aid of Mathematica [8] we obtain Fig. 2, here, for comparison we also depict the graphs of the functions $E_{1}=32\left(\rho_{B} / \rho_{0}\right)^{0.7}$ and $E_{2}=32\left(\rho_{B} / \rho_{0}\right)^{1.1}$.

It is easily verified that $E_{\text {sym }}\left(\rho_{B}\right)$ with graph given in Fig. 2 can be approximated by the function

$$
E_{\text {sym }} \approx 32\left(\rho_{B} / \rho_{0}\right)^{1.05} .
$$




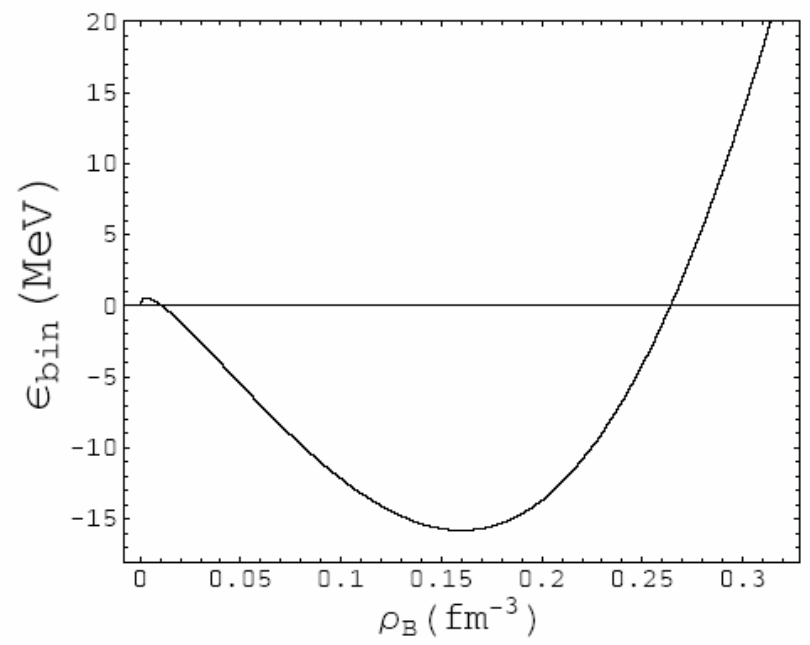

Fig. 1. The $\rho_{B}$ dependence of $\epsilon_{b i n d}$ in symmetric nuclear matter.

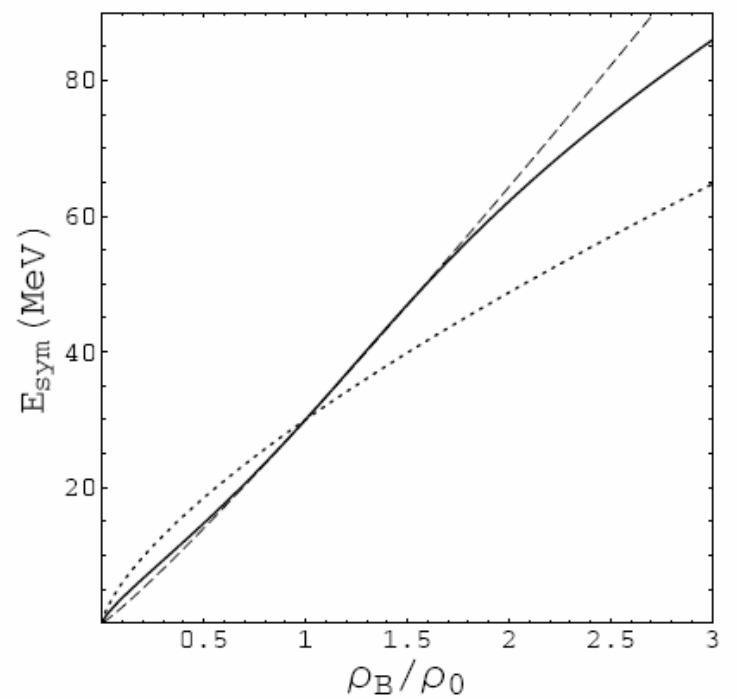

Fig. 2. The $\rho_{B} / \rho_{0}$ dependence of $E_{s y m}$ (solid line), $E_{1}$ (dotted line) and $E_{2}$ (dashed line).

The preceding expression for NSE is clearly in agreement with the analysis of Ref. $[9,10,11]$.

To proceed further let us go to the isobaric incompressibility of ANM, which at saturation density can be expanded around $\alpha=0$ to second order in $\alpha$ as [12]

$$
K(\alpha) \approx K_{0}+K_{a s y} \alpha^{2} .
$$


with $K_{\text {asy }}$ being the isospin-dependent part [13]

$$
K_{\text {asy }} \approx K_{\text {sym }}-6 L \text {. }
$$

$K_{a s y}$ can be extracted from experimental measurements of giant monopole resonances in neutronrich nuclei. $K_{0}$ is incompressibility of symmetric nuclear matter at $\rho_{0}$.

In the following are given respectively the computed values of parameters directly connected with NSE:

- The slop parameter $L=105.997 \mathrm{MeV}$ which is consistent with the result of Ref.[14].

- The symmetry pressure $P_{\text {sym }}=\rho_{0} L / 3=4.3410^{7} \mathrm{MeV}^{4}=0.0286 \mathrm{fm}^{-4}$ which is very useful for structure studies of nuclei.

- $K_{a s y}=-549.79 \mathrm{MeV}$. This value is in good agreement with another works [14, 15].

- $K_{0}=547.56 \mathrm{MeV}$.

\section{CONCLUSION}

Developing the previous work [6] we have carried out in this paper a more realistic study concerning isospin degree of freedom of ANM. The equation of state of ANM given in (12) is our principal result. The DBA was used to compute numerically the density dependence of NSE and other physical quantities of ANM. The obtain results are quite consistent with recent works, except for $K_{0}$, which is too large. This is the shortcoming of the present model. It is evident that EOS of ANM is a fundamental issue for both nuclear physics and astrophysics. It governs phase transitions in ANM. However, we should bear in mind the fact that phase transitions are basically non-perturbative phenomena. Therefore, in this research domain we really need a nonperturbative approach. It is our EOS which was obtained by means of the CJT effective action formalism, a famous non-perturbative method of quantum field theory, and, as a consequence, it could be most suitable for the study of phase transitions and other nuclear properties beyond mean field approximation.

\section{ACKNOWLEDGMENT}

This paper is supported by the Vietnam National Foundation for Science and Technology Development.

\section{REFERENCES}

[1] V.E.Viola, Nucl. Phys. A734 (2004) 487; Phys. Rept. 434 (2006) 1 and references herein.

[2] V. A. Karnaukhov et al., Phys. Rev. C67 (2003) 011601; Nucl. Phys. A734 (2004) 520.

[3] H. Mueller and B. D. Serot, Phys. Rev. C52 (1995) 2072.

[4] M. Malheiro, A. Delfino, and C. T. Coelho, Phys. Rev. C58 (1998) 426.

[5] J. Richert and P. Wagner, Phys. Rept. 350 (2001) 1 and references herein.

[6] Tran Huu Phat, Nguyen Tuan Anh, Nguyen Van Long, and Le Viet Hoa, Phys. Rev. C76 (2007) 045202.

[7] J. Cornwall, R. Jackiw and E. Tomboulis, Phys. Rev. D10 (1974) 2428.

[8] Wolfram, Stephen: The Mathematica Book, Fifth Edition. Wolfram Media/Cambridge University Press, 2003.

[9] B. A. Li, L. W. Chen, C. M. Ko and A. W. Steiner, nucl-th/0601028.

[10] B. A. Li and L. W. Chen, Phys. Rev. C72 (2005) 064611.

[11] L. W. Chen, C. M. Ko and B. A. Li, Phys. Rev. Lett. 94 (2005) 032701.

[12] M. Prakash and K. S. Bedell, Phys. Rev. C32 (1985) 1118.

[13] V. Baran, M. Colonna, M. Di Toro, V. Greco, M. Zielinska-Pfabe and M. H. Wolter, Nucl. Phys. A703 (2002) 603.

[14] L. W. Chen, C. M. Ko, and B. A. Li, Phys. Rev. Lett. 94 (2005) 032701.

[15] T. Li et al., Nucl-ex/0709.0567.

Received 15 December 2010. 